\title{
EVALUATION OF BONE REGENERATION OF MAXILARY CYSTIC DEFECT GRAFTED WITH PUERARIN WITH CALCIUM SULPHATE GRANULES VERSUS CALCIUM SULPHATE AS A SOLOGRAFT: A RANDOMIZED CLINICAL TRIAL
}

\author{
Saleh Ahmed Bakry* and Hesham Fattouh*
}

\begin{abstract}
Aim: The aim of this study was to evaluate bone regeneration of maxillary cystic defect grafted with puerarin with calcium sulphate granules versus calcium sulphate granules as a solograft.

Materials and Methods: This was a randomized controlled clinical trial conducted on 20 patients suffering from maxillary cystic lesions with size more than $3 \mathrm{~cm}^{2}$ indicated for enucleation without the need for resection or plate reconstruction.

In the first group (A): Bony cavities were grafted by Puerarin mixed with hemihydrated calcium sulphate bone graft granules, while in the second group (B): The bony cavities were grafted by hemihydrated calcium sulphate bone graft granules only. All patients were followed up for 6 months recording the progress of the healing both clinically and radiographically via CBCT.

Results: Surgeries went uneventful in patients of both groups. No notable complications occurred during the surgical procedures and the healing period of the two groups. Radiographic results after 6 months showed that there was a significant decrease in cyst volume in the purerein group compared to the other group.
\end{abstract}

Conclusions: Puerarin is a promising graft material with probably an osteoinductive role, an issue that needs more researches to optimize its use and to understand its bone forming mechanism.

KEYWORDS: Puerarin, calcium sulphate, cyst enucleation, graft material.

\section{INTRODUCTION}

The oral cyst can be defined as pathological cavities that may contain air, fluids, or semi-solid material. ${ }^{(1-3)}$ The bones with the highest count of cysts in the human body are the bone of the jaws. This is due to the abundant amount of epithelial remnants that can be left in the bones of the jaws during odontogenesis (tooth development). This resting epithelium is usually dormant or undergoes atrophy, but, when stimulated, they may form a cyst. ${ }^{(1-5)}$

\footnotetext{
* Associate Professor Oral Surgery Department, Faculty of Dentistry, Cairo University, Egypt
} 
The reasons why resting epithelium may proliferate and undergo cystic transformation are generally unknown, but inflammation is assumed to be a serious factor. The high rate of impacted tooth and dental infections that occur within the bones of the jaws is a significant to elucidate why cysts are more common at these sites. ${ }^{(3-5)}$

The most common type of inflammatory odontogenic cysts of the jaws is the radicular cyst which consist nearly $65 \%-70 \%$ of all jaw cysts, and dentigerous cyst is the most common type of developmental odontogenic cyst of the jaw which is nearly $15 \%-18 \%$ of all jaw cysts. ${ }^{(6)}$

Treatment of odontogenic cysts include conservative endodontic treatment, enucleation, marsupialization, decompression and extraction. In small size cyst defect (less than $2 \mathrm{~cm}$ ) spontaneous regeneration of bone can be occur without the need of any bone graft ${ }^{(7)}$ but in case of large defect clot breakdown and infection are more likely to occur leading to a large hematoma. To overcome these complications of enucleation of large sized cysts and to accelerate the bone healing, oral and maxillofacial surgeons obliterate the dead space of a sizable cyst defect by packing the cavity with bone or a bone substitute. The variety of grafts used is autogenous, allogenous, xenografts or alloplastic materials. Autogenous bone is the gold standard in grafting procedures for its osteogenic, osteoinductive properties, however autograft has limitations, including donor site morbidity, insufficient amount, and inappropriate form. These limitations have prompted increasing interest in alternative to bone grafts. Nevertheless, most bone substitutes lack the osteogenic, osteoinductive properties of autogenous bone ${ }^{(8-12)}$

Phytoestrogens are a special class of plant-derived molecules known with this nomenclature because of their partial estrogen agonism with estrogen receptors in mammalian systems. Phytoestrogens present in many fiber rich foods such as berries, seeds, grains, nuts, and fruits. Most phytoestrogens are phenolic compounds like isoflavones. ${ }^{(13)}$

Puerarin is the major bioactive ingredient extracted from the root of Pueraria lobata, which is well known as Gegen in traditional Chinese medicine. Gegen is a medicinal homologous plant with a great history in China. Since then, the pharmacological effects of puerarin have been extensively known and investigated. Puerarin has a wide range of pharmacological properties, which has been widely used in the treatment of diabetes, cardiovascular and cerebrovascular diseases, osteonecrosis, Parkinson's disease, Alzheimer's disease, endometriosis, and cancer However, up till now despite having broad pharmacological activities, the clinical applications of puerarin are limited. According to the database of China Food and Drug Administration, intravenous injections and eye drops seem to be the only drug delivery methods of puerarin ${ }^{(14,15)}$.

Many studies were performed to evaluate the stimulatory effect of puerarin on bone formation and its effect to regenerate the bone. In 2007, Wong et al studied the use of Puerarin as a bone grafting material locally using nine New Zealand white rabbits with 18 bone defects. The defects were divided into three groups. The first group grafted with Puerarin solution mixed with collagen matrix. The second group grafted with collagen matrix alone, while the third group left empty. Results showed $54 \%$ increase in bone regeneration in the first group more than other groups. ${ }^{(16)}$

Calcium sulfate (CS) is well-tolerated when used to fill bone defects and undergoes rapid and complete resorption without eliciting a significant inflammatory response. In addition, CS are often used as a vehicle to deliver antibiotics, pharmacologic agents, and growth factors. It has found wide use in orthopedics and dentistry, and has been utilized in a spread of clinical applications, including the periodontal defect repair, the treatment of osteomyelitis, sinus augmentation, and as an adjunct to implant placement. ${ }^{(17,18)}$ 
The aim of this study is to evaluate the regeneration capacity of the Puerarin mixed with calcium sulphate when used as a bone graft in obliteration of maxillary cyst.

\section{PATIENTS AND METHODES}

\section{Study Design}

This study was a randomized controlled clinical trial conducted on 20 patients ( 8 females and 12 males). The study was conducted in Faculty of Dentistry, Cairo University. The Ethics Committee of the Faculty of Dentistry, Cairo University approved the protocol, and a detailed informed written consent including the details of surgery and the possible complications was obtained from all patients. After proper diagnosis the patients selected were suffering from cystic lesions need to be grafted after cyst encluation.

\section{Eligibility Criteria}

The selected patients suffered from maxillary cyst with size more than $3 \mathrm{~cm}^{2}$ indicating for enucleation and did not need resection or plate reconstruction.

The patients included in the study fulfilled the following criteria

\section{Inclusion Criteria:}

- Patient age ranging from 25-55 years.

- Patients with any type of endophytic maxillary cyst.

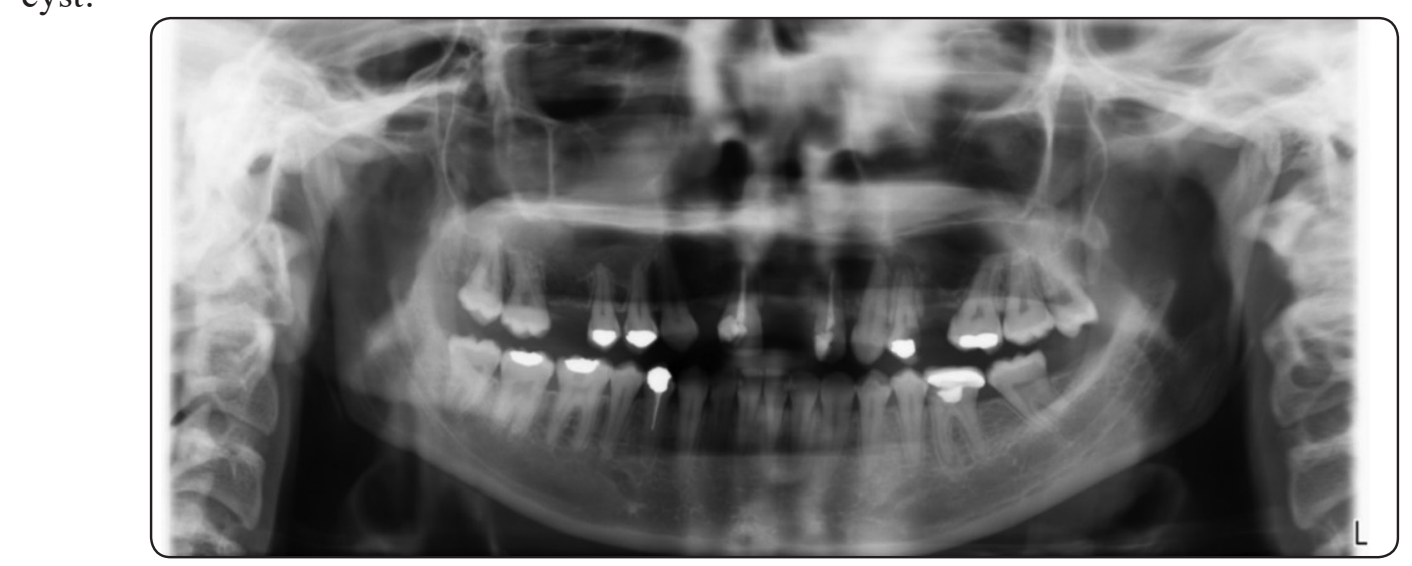

Fig. (1): Panoramic x-ray showed multiple maxillary radiolucent areas suggesting multiple cysts indicated for enucleation
- Cyst size equaled or exceeds $3 \mathrm{~cm}^{2}$.

- Chosen cysts for studying liable for enucleation and did not need resection or plate reconstruction.

\section{Exclusion criteria}

- Patients with any systemic condition that could contraindicate the surgical procedure or interfere with normal bone.

- Large cysts indicating reconstruction.

- Previously operated sites of the maxilla.

- Any infected cyst.

\section{Randomization}

This study was a randomized clinical controlled trial. Patients were randomly assigned into two equal groups: group (A) and group (B) according to the website (http//www.random.org.eg). Each group consisted of 10 patients.

\section{Preoperative Preparation}

At the initial visit, all patients underwent a clinical and dental history to avoid any of the exclusion criteria mentioned above. Digital panoramic radiographs were obtained for evaluating the lesion (Figure 1). The patients were mainly suffering from pain, tenderness and swelling related to the area of interest, most of the patients were claiming pain related to old endodontically treated teeth, or pain and tenderness and swelling around the area of old trauma. Palpation, inspection and percussion were 
the main methods used to examine the patient intraorally where the main signs and symptoms of interest to be found were redness, swelling and tenderness of the area of interest and in some cases mobility of the tooth related to the lesion. Aspiration of the cystic swelling by $10 \mathrm{~cm}$ syringe inserted to the main bulk and aspirated from the lesion in many directions.

\section{Patient grouping}

The selected patients fulfilled the inclusion criteria and randomly allocated to two groups.

\section{Group A (Puerarin group)}

This group consists of ten cases ( 3 females and 7 males) in which surgical inoculation of the cystic lesions took place, the bony cavities was grafted by Puerarin mixed with hemihydrated calcium sulphate bone graft granules (G 170 ghimas s.p.a Italy) as a carrier.

\section{Group B (hemihydrated calcium sulphate group):}

This group consists of ten cases (5 females and 5 males) in which surgical inoculation of the cystic lesions took place, the bony cavities was grafted by hemihydrated calcium sulphate bone graft granules only (G 170 ghimas s.p.a Italy).

\section{Surgical method}

Before starting of the surgical operation any tooth of mobility grade 2 or more was extracted, all teeth related to the lesion were endodontically treated, scaling was done and the patient was instructed to use Chlorohexidine Gluconate $0.1 \%$ mouth wash (Antiseptol, Kahira Pharma Co, Cairo, Egypt.) before surgery to maintain the oral hygiene. All surgical procedures were performed under local anesthesia (Articane $4 \%$ with 1: 100 000 epinephrine, UbistesinTM forte, 3M ESPE, Germany.). At time of surgery, the peri-oral area was scrubbed with antiseptic solution Povidone iodine (Betadin, Nile, Egypt). The oral cavities were rinsed with chlorhexidine mouth rinse (Antiseptol, Kahira
Pharma Co, Cairo, Egypt.) to obtain an aseptic environment. A mucoperiosteal full thickness 3 wall flaps were reflected using a mucoperiosteal elevator. Bone removal was performed using surgical fissure bur and bone rongeure end and side cutting according to the thickness of the covering bone. After exposure of the cyst lining the cyst was enucleated with bone curette, the root canal treated teeth were apicectomied and retro grade filled using Mineral trioxide aggregate (MTA). The undermined bone of the surgical defect was removed, the surgical cavity was debrided and irrigated with saline.

For group A, Puerarin was mixed with hemihydrated calcium sulphate bone graft granules (G 170 ghimas s.p.a Italy) and the mixture was used for augmentation of the cystic cavity (Figure 2). For group B the bony cavities were grafted by hemihydrated calcium sulphate bone graft granules only (Figure 3) (G 170 ghimas s.p.a Italy).

Flaps were suture by $3 / 0$ black silk suture, and post-operative medication and instructions were prescribed to the patients. Postoperatively, the patients were instructed to use extraoral ice packs for the first postoperative six hours. The gauze pad placed over the surgical area should be kept in place for a half hour. After this time, the gauze pad should be removed and discarded. Vigorous mouth rinsing or touching the wound area following surgery should be avoided. This may initiate bleeding by causing the blood clot that has formed to become dislodged. Non-steroidal anti-inflammatory analgesic (Diclofenac potassium 50mg, Catafast $50 \mathrm{mg}$ tablets, Novartis Pharma AG, Cairo, Egypt.) was prescribed three times daily for three days. Antibiotic (clindamycin $300 \mathrm{mg}$, Clindam $300 \mathrm{mg}$ capsules, Sigma pharmaceutical industries, Egypt) was prescribed three times daily for five days. The mouth wash was started 24 hours after surgery 3 times a day for 1 week.

The patients were recalled to remove the sutures after 7 days and to ensure that they are free of any post-operative complications as fenestration, 


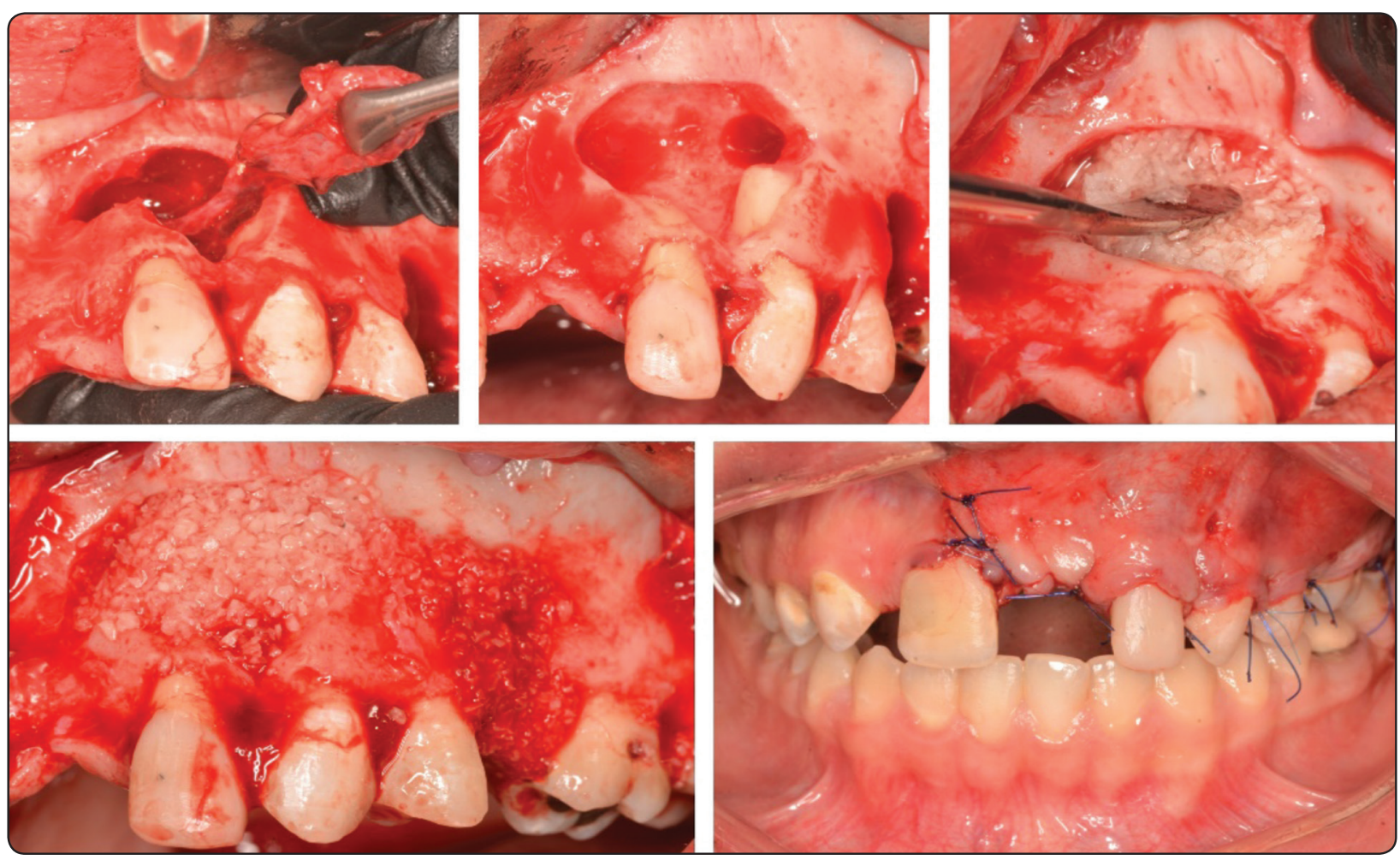

Fig. (2): Clinical pictures for group A showing the removal of the cyst lining and the augmentation of the cystic cavity by Puerarin mixed with hemihydrated calcium sulphate bone graft granules.

breakage of the sutures, loss of the graft or infection at the site of interest which threatens the healing. Patients were recalled after one week and 6 months of the surgery to perform each time a CBCT for follow-up of the progress of the healing. Data from CBCT were exported and transferred in DICOM format for volumetric assessment. After importing data as DICOM files into the software, a series of steps were followed to create a mask for the bone defect, where the volume of the defect was compared at the series of scans. A thresholding was made to accommodate the density of the defect, and then a crop tool was utilized to adjust the threshold mask to the defect area only. After editing the defect for any discrepancy in shape, the volume was then calculated by creating a 3D mask (Figure 4-6). Visual analogue score graded from zero - 10 was used to estimate sever pain for each patient from the two groups considering 7:10 for sever pain.
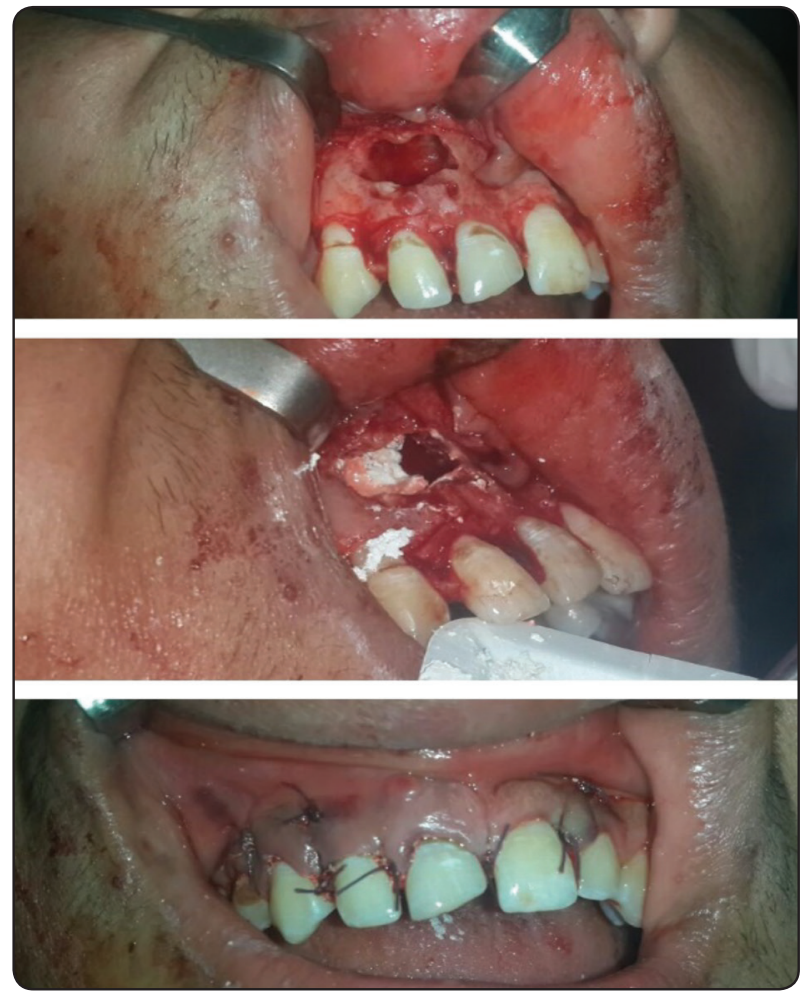

Fig. (3) Clinical pictures for group B showing the removal of the cyst lining and the augmentation of the cystic cavity by hemihydrated calcium sulphate bone graft granules 


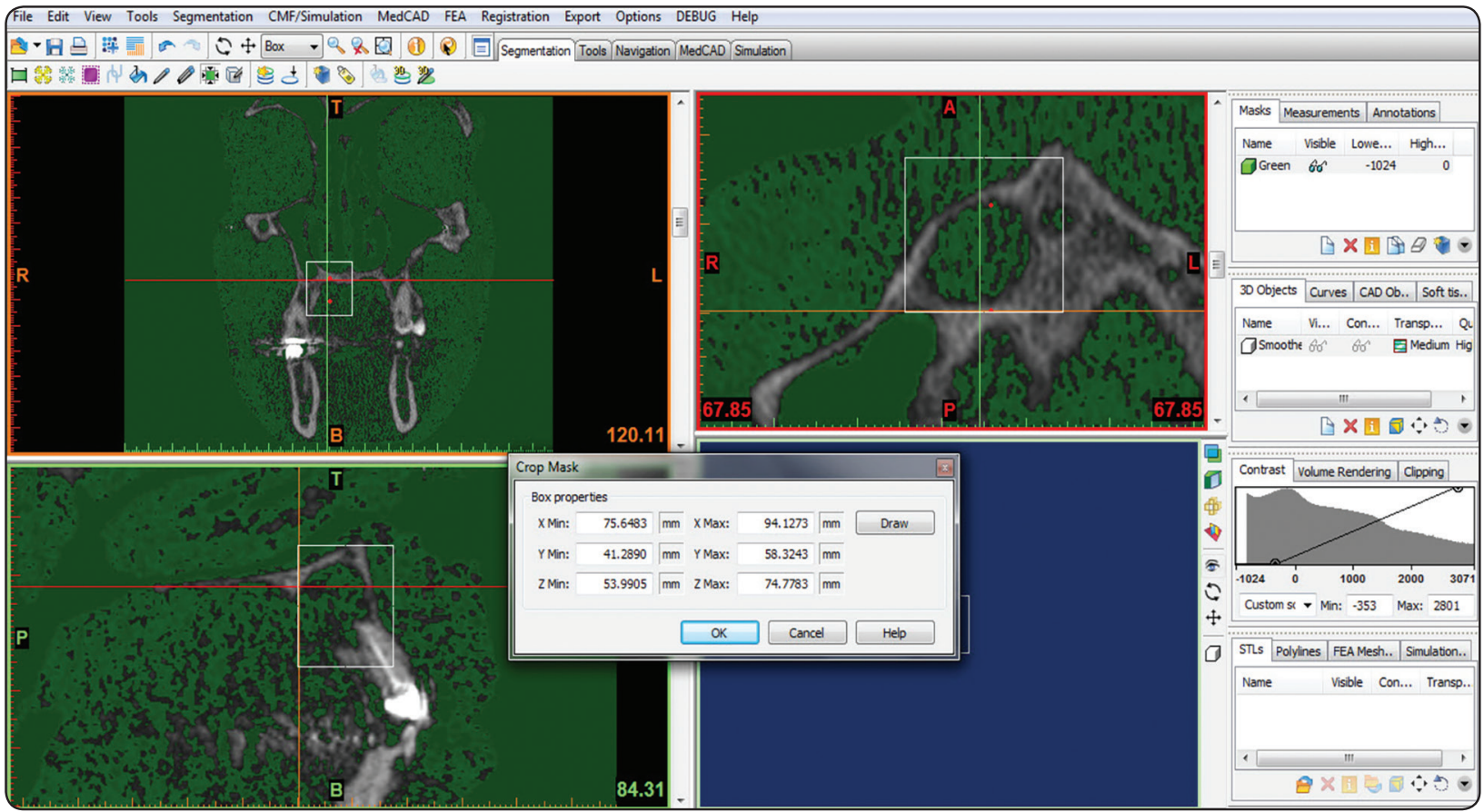

Fig. (4) Measurement of the dimensions of the defect preoperatively

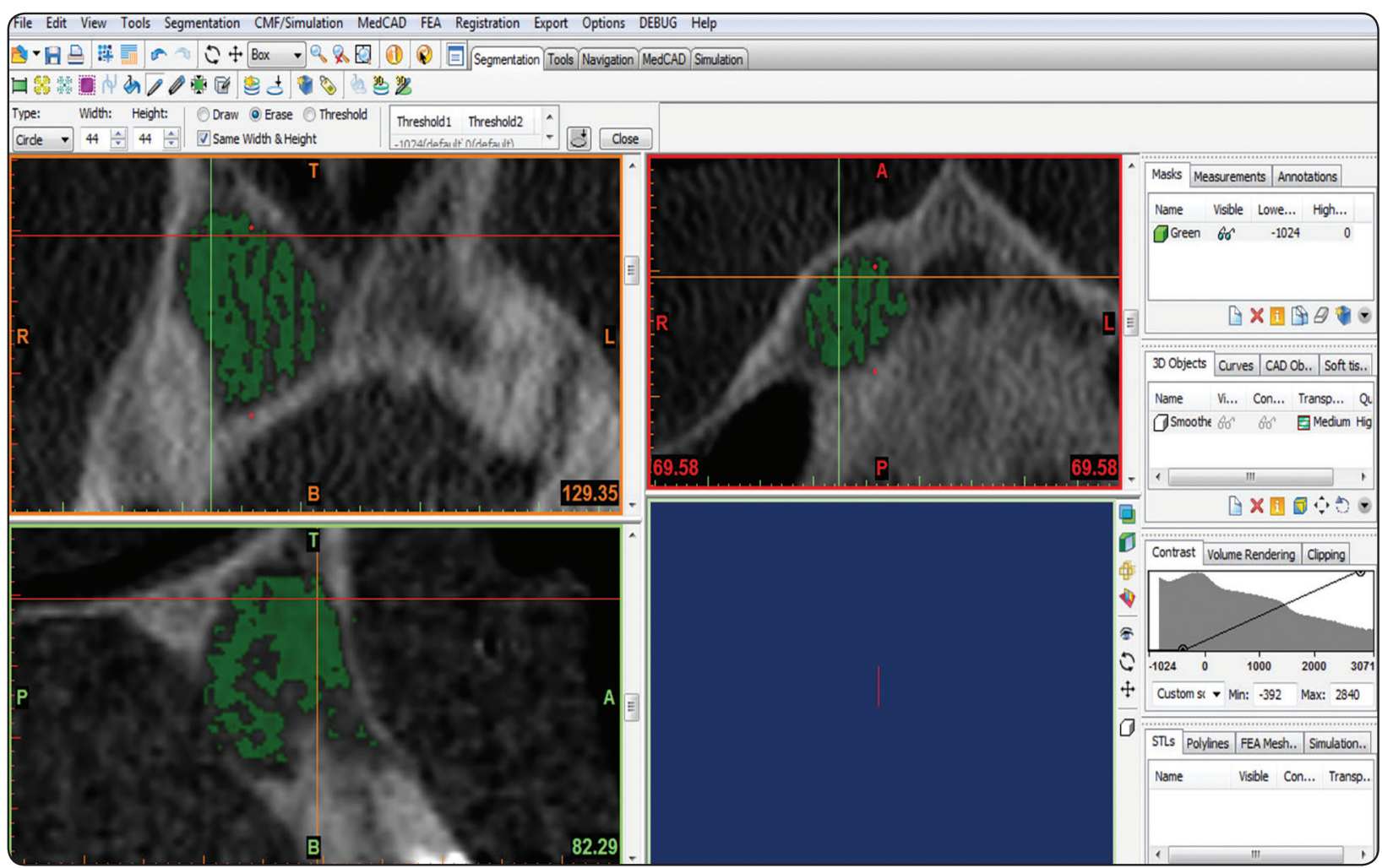

Fig. (5) The reference points for measurement that will be taken in consideration during measurement postoperative 


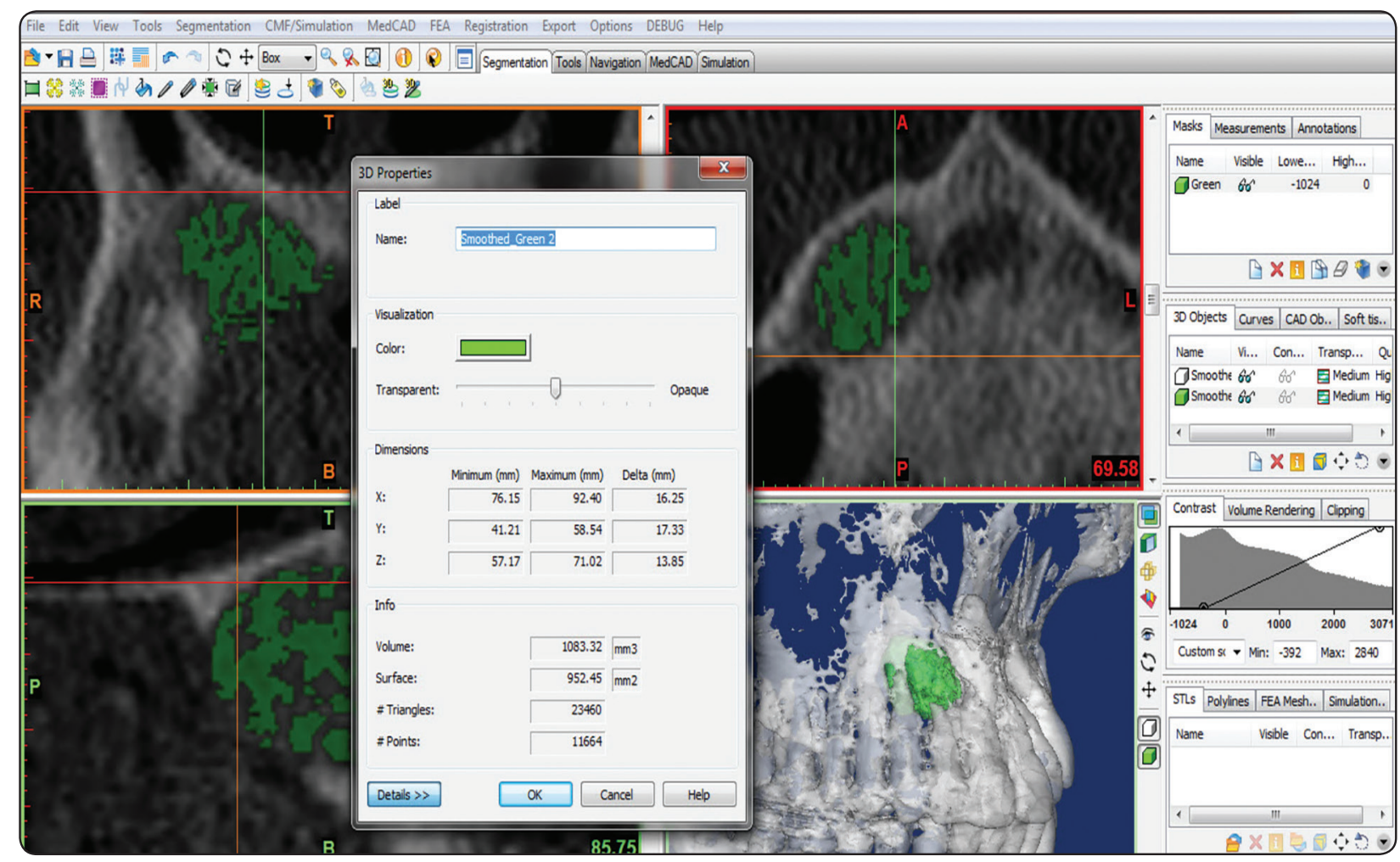

Fig. (6) Measuring the volume of the cavity while healing and the difference of size measured from the reference point

\section{Statistical analysis}

The collected data was analyzed using the Statistical Package for Social Sciences (SPSS) version 22. Numerical data was summarized using means standard deviations and ranges. Categorical data from both groups was summarized as percentages. Comparisons between the two groups were performed using the t-test. For categorical variables, differences were analyzed with Fisher's exact test. P-values $\leq 0.05$ were considered significant to determine the difference between both groups.

\section{RESULTS}

This study was conducted on 20 patients (8 females and 12 males) with endophytic maxillary cyst indicated for enucleation. The patients were divided into 2 groups. The cavity was grafted by Puerarin mixed with hemihydrated calcium sulphate bone graft for group A and hemihydrated calcium sulphate granule bone graft for group B. the mean age was $32.4 \pm 12.4$ years for group A, and $35.6 \pm$ 8.9 years for group B., and there was no statistical difference in the age between the two groups ( $\mathrm{P}$ value 0.52 ). The surgery was uneventful for all the patients. No notable complications occurred during the surgical procedure and the healing period of the two groups. Both groups showed moderate post-operative pain after 1 week. The pain was comparable between the two groups $(5.9 \pm 1.8$ for group A, $5.4 \pm 1.7$ for group B) with no statistical significance difference (P value 0.53). After 1 month the pain significantly declined in the 2 groups $(0.5$ \pm 0.7 for group A, $0.3 \pm 0.5$ for group B) and there was no statistical significance difference in the pain after 1 month between the both groups ( $\mathrm{P}$ value 0.47). For the difference in defect size, there was no significance difference between the defect size in (group A) and the defect size in (group B) 1 week postoperatively (P value 0.95). However, group A 
showed higher decrease in volume after 6 months compared to group B $(51.5 \pm 14.5,39.3 \pm 11, \mathrm{P}$ value 0.048) (table 1 ) and (Figure 7).

TABLE (1) Showing the defect size in millimeters for the two groups at different time points

\begin{tabular}{|c|c|c|c|}
\hline & Group A & Group B & P value \\
\hline 1 week & $261.6 \pm 91.5$ & $258.7 \pm 109.3$ & 0.95 \\
\hline 6 months & $210.1 \pm 80.7$ & $219.4 \pm 102.5$ & 0.82 \\
\hline Change & $51.5 \pm 14.5$ & $39.3 \pm 11$ & $0.048^{*}$ \\
\hline
\end{tabular}

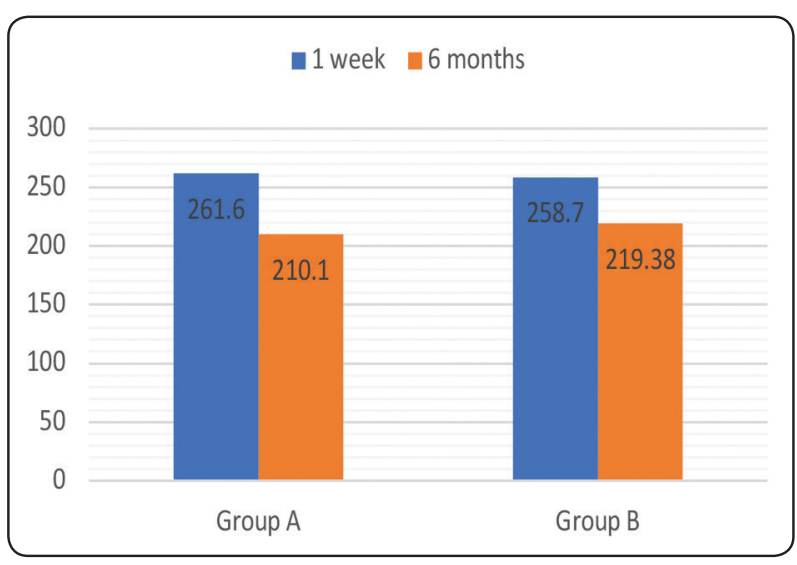

Fig. (7) Bar chart showing the change in the defect size in millimeters for the two groups

\section{DISCUSION}

Odontogenic cysts with diameter of $3 \mathrm{~cm}$ or more are usually treated by careful enucleation and obliterating the defect with different bone potential fillers, defects with these sizes if left without obliteration do not completely and spontaneously heal no matter how long they are observed (19), the unhealing process is usually associated with a great possibility of infection, bone weakening, and pathological fracture may occur. Literature lacks evidence to support one kind of different filling materials over the other ${ }^{(20)}$.
Puerarin is considered as one of the recently evolved bone grafts augmentation material that possesses a wide range of pharmacological properties and has been widely used in the treatment of various conditions. Puerarin is a promising material with a little amount of knowledge we still have about its osteoinduction role in bone repair. ${ }^{(21)}$

In the study group the defects were filled by purerarin with calcium sulphate, while in the control group the defects were filled by calcium sulphate alone. In the control group, calcium sulphate act as a grafting material with well-known osteoconductive prperties, while in the study group it serves as a carrier for the purerarin.

Our results showed significant regeneration capacity of the puerarin as a promising material in that field in comparison to the osteoconductivity of the calcium sulphate. These results agree with Rabie et al. who made a study in 2007 , to observe puerarin effect on bone formation, he intended to make a defect in the parital bone of white rabbits and compared the healing effect of puerarin when mixed with collagen matrix versus collagen matrix alone, the result showed a total $500 \%$ more new bone formation was present in defects grafted with puerarin in collagen matrix than those grafted with the collagen matrix alone ${ }^{(22)}$.

Türer et al. conducted another study to assess the healing potential of local puerarin administration on xenograft in cranial bone defect treatment in rats. They showed statistically higher new bone formation in the puerarin xenograft group compared to the xenograft alone group ${ }^{(23)}$. Puerarin also has been used for bone regeneration in maxillary sinus augmentation in patients with posterior edentulous maxilla and showed new bone formation in sinus augmentation in $100 \%$ of the patients ${ }^{(24)}$.

Calcium sulphate showed inferior regeneration capacity when used alone compared to peurarine calcium sulphate mixture, this comes with what Virendra et al concluded in 2015 , he made a study 
to evaluate bony healing following filling of post cystectomy jaw bone defects with a cyst size less than $5 \mathrm{~cm}$ with various osteoconductive materials compared to a graftless control group, his results showed no significant difference in spontaneous bone regeneration in post cystectomy defects between the grafted groups and the control one ${ }^{(25)}$.

Regarding our radiological assessment, it is well known that the CBCT scan is great diagnostic tool for evaluating the effect of different grafting materials recording the progress of the healing process via monitoring the difference in the defect volume. The present study focused on radiographic changes of bone graft material volume evaluated by CBCT analysis by made a thresholding to accommodate the density of the defect, and then a crop tool was utilized to adjust the threshold mask to the defect area only. This mechanism increases the accuracy of measuring the volume change in the cyst defect.

So, Puerarin is a promising graft material with probably an osteoinductive role, an issue that needs more researches to optimize its use and to understand its bone forming mechanism.

\section{REFRENCES}

1. Charles D. Cysts of the jaws. University of Missouri Kansas City - School of Dentistry. 2017.https://dentistry. umkc.edu/wp/Jaw cysts.pdf.

2. Ghom M. Cysts of the orofacial region. Textbook of oral pathology. 1st Edition. 2010. https://www.researchgate. net/publication.

3. Dardo M. Cysts and pseudocysts of the oral cavity: Revision of the literature and new proposed classification. International Journal of Experimental and Clinical Pathophysiology 2018: Volume 32 - Issue 5: Page 999-1007.

4. Miller-Keane Encyclopedia and Dictionary of Medicine, Nursing, and Allied Health. 7th Edition. Saunders: an imprint of Elsevier. 2015. Cysts: Page 212-214.

5. Joseph R. Oral pathology, Clinical pathologic correlations: Medicine, Internal medicine. 2017. Technologists and Cram Inc. 5th Edition.
6. Stockdale CR, Chandler NP. The nature of the periapical lesion: a review of 1108 cases. J Dent 1988;16: 123-129.

7. Pradel W. Bone regeneration after enucleation of mandibular cysts comparing autogenous grafts from tissue engineered bone. Oral Surgery Oral Medicine Oral Pathology Oral Radiology Journal. 2006. Volume 101 - Issue 3: Page 285-290.

8. Junho L. Comparison of autogenous tooth bone graft and synthetic bone graft materials used for bone resorption around implants after crestal approach sinus lifting: a retrospective study. Journal of periodontal implant society. 2014. Volume 44 Issue 5: Page 216-221.

9. Banwart JC, Asher MA, Hassanein RS. Iliac crest bone graft harvest donor site morbidity: A statistical evaluation. Spine 1995; 20: 1055-60.

10. Cowley SP, Anderson LD. Hernias through donor sites for iliacbone grafts. J Bone Joint Surg Am 1983; 65: 1023-25.

11. Summers BN, Eisenstein SM. Donor site pain from the ilium: a complication of lumbar spine fusion. J Bone Joint Surg Br 1989; 71: 677-80.

12. Albrektsson T. Osteoinduction, osteoconduction and osseointegration. European Spine Journal. 2001. Volume 2 - Issue 2: Page 96-101.

13. Lucia B. Phytoestrogen food or drug? Journal of clinical cases in mineral and bone metabolism. 2007. Volume 4 Issue 2: Page 123-130.

14. Li Y, Ye H, Wang T, Wang P, Liu R, Li Y, Tian Y, Zhang J.Mar. Characterization of Low Molecular Weight Sulfate Ulva Polysaccharide and its Protective Effect against IBD in Mice Drugs. J Ind Eng Chem 2020 Sep 29;18(10):499.

15. Yong-Deok Jeon, Ji-Hyun Lee, Young-Mi Lee, Dae-Ki Kim. Puerarin inhibits inflammation and oxidative stress in dextran sulfate sodium-induced colitis mice model. The Open Orthopedics 2020 Apr; 124:109847.

16. Wong R. Effect of Quercetin on preosteoblasts and bone Defects. The Open Orthopedics Journal. 2008. Volume 2 Issue 1: Page 27-32.

17. Shahida N, Bashah K, Fauzi A, and Noor M. ScienceDirect. The influence of silicon addition in modulation of HA / TCP ratio in biphasic calcium phosphate. Mater Today Proc.2019.16:1796-1803.

18. Shim K, Kim H, Eum S, and Park K. Journal of Industrial and Engineering Chemistry Simple surface biofunctional- 
ization of biphasic calcium phosphates for improving osteogenic activity and bone tissue regeneration. J Ind Eng Chem.2018.68:220-228.

19. Kattimani VS, Bajantai NV, Sriram SK, Sriram RR, Rao VK, Desai PD. Observer strategy and radiographic classification of healing after grafting of cystic defects in maxilla: a radiological appraisal. The journal of contemporary dental practice. 2013 Mar-Apr;14(2):227-32

20. Zicai K. Establishing a critical-size mandibular defect model in growing pigs characterization of spontaneous healing. Journal of Oral and Maxillofacial Surgery. 2014. Volume 72 - Issue 9: Page 52-68

21. Zhou YX, Zhang H, Peng C. (2014). Puerarin: a review of pharmacological effects. Phytother Res 28:961-75.
22. Rabie B. Effect of puerarin on bone formation. Osteoarthritis and Cartilage Journal. 2007. Volume 15 - Issue 8: Page 894-899.

23. Türer A, Türer ÇC, Önger ME. Effect of Local Puerarin Administration on Xenograft. J Clin Anal Med 2017;8(5): 396-400.

24. Khashaba M. Evaluation of the Effect of Puerarin on Bone Regeneration in Maxillary Sinus Augmentation (MD thesis). Cairo University. 2013

25. Virendra K. Bony healing following filling of post cystectomy jaw bone defects with hydroxyapatite and Tricalcium phosphate and its comparison with non-filling case. International Journal of Scientific Study. 2015. Volume 3 - Issue 9: Page 102-106 